\title{
A REMARK ON THE DIOPHANTINE EQUATION
}

$$
\left(x^{3}-1\right) /(x-1)=\left(y^{n}-1\right) /(y-1)
$$

Bo $\mathrm{He}$

Sichuan Normal University, China

\footnotetext{
ABSTRACT. In this remark, we use some properties of simple continued fractions of quadratic irrational numbers to prove that the equation

$$
\frac{x^{3}-1}{x-1}=\frac{y^{n}-1}{y-1}, x, y, n \in \mathbb{N}, x>1, y>1, n>3,2 \nmid n
$$

has only the solutions $(x, y, n)=(5,2,5)$ and $(90,2,13)$.
}

For any positive integer $N$ with $N>2$, let $s(N)$ denote the number of solutions $(x, m)$ of the equation

$$
N=\frac{x^{m}-1}{x-1}, x, m \in \mathbb{N}, x \geq 2, m>2 .
$$

Ratat [17] in 1916 and Goormaghtigh [10] in 1917 found that $s(31)=2$ and $s(8191)=2$, respectively. We consider the equation

(2) $\frac{x^{m}-1}{x-1}=\frac{y^{n}-1}{y-1}, x>1, y>1, m>2, n>2, x \neq y$, for $x, y \in \mathbb{N}$.

It has been conjectured that the equation (2) has only a finite number of solutions, even that has only two solutions $(x, y, m, n)=(5,2,3,5),(90,2,3,13)$.

This is rather a difficult question. Many authors have proved that if two of the variables $x, y, m, n$ are fixed then the equation (2) has a finite number of solutions. See for examples $[1,3,4,5,12,13,19,20,21,16,22,23,24]$. Remark that two known solutions of (2) are both satisfying $m=3$. If $m=3$, the equation (2) has the form

$$
\frac{x^{3}-1}{x-1}=\frac{y^{n}-1}{y-1}, x, y, n \in \mathbb{N}, x>y>1, n>3 .
$$

2000 Mathematics Subject Classification. 11A55, 11D09, 11D61.

Key words and phrases. Goormaghtigh equation, continued fraction, congruence. 
We know that the equation (3) has two solutions $(x, y, n)=(5,2,5)$ and $(90,2,13)$, and any other possible solution is called an exceptional solution [13]. If we prove that (3) has no exceptional solutions, then the conjecture is true under the condition $m=3$. Le [12] proved that (3) has no exceptional solution with $\omega(y)>1$, where $\omega(a)$ denote the number of distinct prime divisors of $a$ (the reference [12] contains an error, one can refer to [2] for a correct version). Nesterenko and Shorey [16] proved that any exceptional solution of (3) with $2 \nmid n$ must be $n \geq 25$. Le [14] has given the relative upper bound, namely, $x<2^{\left(n^{2}-4 n+6\right) / 2}$ and $y<2^{(n-3) / 2}$.

In [13], Le proved that, for any exceptional solution of (3), we must have $\operatorname{gcd}(x, y)>1$ and $y \nmid x$. In 2005, Yuan [26] used this result and properties of Pellian equations and proved the following result.

ThEOREM 1. The equation (3) has only the solutions $(x, y, n)=(5,2,5)$ and $(90,2,13)$ with $n$ is odd.

In this paper, we prove Theorem 1 using another method. We will use the simple continued fraction expansion to express the solutions of the Pellian equation obtained from (3), and we get a contradiction to the result in [13] by congruence relations.

Now, let us recall some properties of continued fractions. The simple continued fraction expansion of a quadratic irrational $\alpha=\frac{a+\sqrt{d}}{b}$ is periodic. This expansion can be obtained using the following algorithm [11]. Let $s_{0}=$ $a, t_{0}=b$ and

$$
a_{k}=\left\lfloor\frac{s_{k}+\sqrt{d}}{t_{k}}\right\rfloor, \quad s_{k+1}=a_{k} t_{k}-s_{k}, \quad t_{k+1}=\frac{d-s_{k+1}^{2}}{t_{k}}, \quad k \geq 0 .
$$

If $\left(s_{c}, t_{c}\right)=\left(s_{d}, t_{d}\right)$ for $c<d$, then

$$
\alpha=\left[a_{0}, \ldots, a_{c-1}, \overline{a_{c}, \ldots, a_{d-1}}\right] .
$$

Let $p_{n} / q_{n}$ denote the $n^{\text {th }}$ convergent of $\alpha$. The following result of Worley [25] and Dujella [6] extends classical results of Legendre and Fatou [9] concerning Diophantine approximations of the form $\left|\alpha-\frac{a}{b}\right|<\frac{1}{2 b^{2}}$ and $\left|\alpha-\frac{a}{b}\right|<\frac{1}{b^{2}}$.

Lemma 2 (Worley [25], Dujella [6]). Let $\alpha$ be a real number and $a$ and $b$ coprime nonzero integers, satisfying the inequality

$$
\left|\alpha-\frac{a}{b}\right|<\frac{\sigma}{b^{2}}
$$

where $\sigma$ is a positive real number. Then $(a, b)=\left(r p_{k+1} \pm u p_{k}, r q_{k+1} \pm u q_{k}\right)$, for some $k \geq-1$ and nonnegative integers $r$ and $u$ such that $r u<2 \sigma$.

In fact, by Fatou [9] we have

$$
\frac{a}{b}=\frac{p_{k}}{q_{k}} \text { or } \frac{p_{k+1} \pm p_{k}}{q_{k+1} \pm q_{k}}
$$


for $\sigma=1$. And explicit versions of above result for $\sigma=2$, were given by Worley [25, Corollary, p. 206]: $\left|\alpha-\frac{a}{b}\right|<\frac{2}{b^{2}}$, implies

(6) $\frac{a}{b}=\frac{p_{k}}{q_{k}}, \frac{p_{k+1} \pm p_{k}}{q_{k+1} \pm q_{k}}, \frac{2 p_{k+1} \pm p_{k}}{2 q_{k+1} \pm q_{k}}, \frac{3 p_{k+1}+p_{k}}{3 q_{k+1}+q_{k}}, \frac{p_{k+1} \pm 2 p_{k}}{q_{k+1} \pm 2 q_{k}}$ or $\frac{p_{k+1}-3 p_{k}}{q_{k+1}-3 q_{k}}$.

For the explicit results of the bigger $\sigma$, please refer [7].

The next useful result is due to Dujella and Jadrijević [8]. It helps us to simplify our proof.

LEMMA 3. Let ab be a positive integer which is not a perfect square, and let $\frac{p_{k}}{q_{k}}$ denotes the $k^{\text {th }}$ convergent of continued fraction expansion of $\sqrt{\frac{a}{b}}$. Let the sequences $\left(s_{k}\right)$ and $\left(t_{k}\right)$ be defined by $(4)$ for the quadratic irrational $\frac{\sqrt{a b}}{b}$. Then

$$
a\left(r q_{k+1}+u q_{k}\right)^{2}-b\left(r p_{k+1}+u p_{k}\right)^{2}=(-1)^{k}\left(u^{2} t_{k+1}+2 r u s_{k+2}-r^{2} t_{k+2}\right) .
$$

The following lemma is due to Le [13].

LEMma 4. If $(x, y, n)$ is a exceptional solution of equation (3), then $\operatorname{gcd}(x, y)>1$ and $y \nmid x$.

Proof of Theorem 1. Let $(x, y, n)$ be a solution of (3) with $n$ odd. Let us rewrite (3) into

$$
(y-1)(2 x+1)^{2}-4 y\left(y^{(n-1) / 2}\right)^{2}=-3 y-1, \quad n>3 .
$$

Let $\operatorname{gcd}(2 x+1, y)=d$. Then $d$ is a divisor of $-3 y-1$. This implies $d=1$, since $\operatorname{gcd}(-3 y-1, y)=1$. Now, assume that $y \geq 2$. Let us put $X=2 x+1$ and $Y=y^{(n-1) / 2}$ with $\operatorname{gcd}(X, Y)=1$. Then we have

$$
\begin{aligned}
\left|\sqrt{\frac{y-1}{4 y}}-\frac{Y}{X}\right| & =\left|\frac{y-1}{4 y}-\frac{Y^{2}}{X^{2}}\right| \cdot\left|\sqrt{\frac{y-1}{4 y}}+\frac{Y}{X}\right|^{-1} \\
& <\frac{3 y+1}{4 y X^{2}} \cdot\left|2 \sqrt{\frac{y-1}{4 y}}\right|^{-1}=\frac{3 y+1}{4 \sqrt{y(y-1)}} \cdot X^{-2} .
\end{aligned}
$$

It follows that

$$
\left|\sqrt{\frac{y-1}{4 y}}-\frac{Y}{X}\right|<\frac{\sigma}{X^{2}}
$$

where $\sigma=1$ if $y \geq 4$ and $\sigma=2$ if $y=2$ or 3 .

On the other hand, let $\alpha=\sqrt{\frac{y-1}{4 y}}=\frac{\sqrt{4 y(y-1)}}{4 y}$, one can see that

$$
\begin{gathered}
\alpha=[0,2, \overline{y-1,4}], \\
\left(s_{0}, t_{0}\right)=(0,4 y),\left(s_{1}, t_{1}\right)=(0, y-1), \\
\left(s_{2}, t_{2}\right)=(2 y-2,4),\left(s_{3}, t_{3}\right)=(2 y-2, y-1),\left(s_{4}, t_{4}\right)=(2 y-2,4) .
\end{gathered}
$$

Since the period of continued fraction expansion of $\alpha$ is equal to 2, according to Lemma 2, we only need to consider $(X, Y)=\left(r q_{k+1} \pm u q_{k}, r p_{k+1} \pm u p_{k}\right)$ 
for $k=0,1,2$. We use Lemma 3 to check all possibilities $(k, r, \pm u)$ such that the equation

$$
(y-1) X^{2}-4 y Y^{2}=\gamma
$$

satisfies the inequality (8). Thus we have $\gamma \in\{-4, y-1,-3 y-1,5 y-9\}$ for $y \geq 4$ and $\gamma \in\{-4, y-1,-3 y-1,-4 y, 5 y-9,-7 y-9,9 y-25,-11 y-$ $25,12 y-16,13 y-49\}$ for $2 \leq y \leq 3$. Moreover, the result $\gamma=-3 y-1$ comes from

$(k, r, \pm u)= \begin{cases}(2 t, 1,-1),(2 t-1,1,1), & \text { if } y \geq 4, \\ (2 t, 1,-1),(2 t-1,1,1),(2 t, 1,-3),(2 t-1,3,1), & \text { if } 2 \leq y \leq 3 .\end{cases}$

- The cases $(r, \pm u)=(1,1)$ or $(1,-1)$ imply

$$
\left(2 x+1, y^{(n-1) / 2}\right)=\left(q_{2 t+1}-q_{2 t}, p_{2 t+1}-p_{2 t}\right),
$$

or

$$
\left(2 x+1, y^{(n-1) / 2}\right)=\left(q_{2 t}+q_{2 t-1}, p_{2 t}+p_{2 t-1}\right) .
$$

By simple computations, we get

$$
\begin{gathered}
q_{0}=1, \quad q_{2}=2 y-1, \quad q_{2 t+4}=(4 y-2) q_{2 t+2}-q_{2 t}, \\
q_{1}=2, \quad q_{3}=8 y-2, \quad q_{2 t+3}=(4 y-2) q_{2 t+1}-q_{2 t-1} .
\end{gathered}
$$

Then by induction one can easily prove the following property:

$$
q_{2 t} \equiv(-1)^{t} \quad(\bmod 2 y) \text { and } q_{2 t+1} \equiv 2(-1)^{t} \quad(\bmod 2 y) .
$$

From (10), (11) and (12), we get

$$
x \equiv 0 \text { or }-1 \quad(\bmod y) .
$$

But this and Lemma 4 give a contradiction.

- The additional cases $(r, \pm u)=(3,1)$ or $(1,-3)$ (for $y=2,3)$ gives

$$
\left(2 x+1, y^{(n-1) / 2}\right)=\left(q_{2 t+1}-3 q_{2 t}, p_{2 t+1}-3 p_{2 t}\right),
$$

or

$$
\left(2 x+1, y^{(n-1) / 2}\right)=\left(3 q_{2 t}+q_{2 t-1}, 3 p_{2 t}+p_{2 t-1}\right) .
$$

We use a similar argument to get

$$
x \equiv 0 \text { or }-1 \quad(\bmod y) .
$$

We get the contradiction as in the above case.

This completes the proof.

ACKNOWLEDGEMENTS.

The author heartiest thanks Professor Alain Togbé for many helpful suggestions and comments during the preparation of this paper. The author expresses his gratitude to the anonymous referee for constructive suggestions 
to improve an earlier draft of this paper. The author is supported by the Natural Science Foundation of Education Department of Sichuan Province.

\section{REFERENCES}

[1] A. Baker, Bounds for the solutions of the hyperelliptic equation, Proc. Cambridge Philos. Soc. 65 (1969), 439-444.

[2] Y. Bugeaud, T. N. Shorey, On the number of solutions of the generalized RamanujanNagell equation, J. Reine Angew. Math. 539 (2001), 55-74.

[3] Y. Bugeaud, T. N. Shorey, On the diophantine equation $\frac{x^{m}-1}{x-1}=\frac{y^{n}-1}{y-1}$, Pacific J. Math. 207 (2002), 61-75.

[4] J. Coates, An effective p-adic analogue of a theorem of Thue II: The greatest prime factor of a binary form, Acta Arith. 16 (1969/1970), 399-412.

[5] H. Davenport, D. J. Lewis, and A. Schinzel, Equations of the form $f(x)=g(y)$, Quart. J. Math. Oxford Ser. (2) 12 (1961), 304-312.

[6] A. Dujella, Continued fractions and RSA with small secret exponent, Tatra Mt. Math. Publ. 29 (2004), 101-112.

[7] A. Dujella and B. Ibrahimpašić, On Worley's theorem in Diophantine approximations, Ann. Math. Inform. 35 (2008), 61-73.

[8] A. Dujella and B. Jadrijević, A family of quartic Thue inequalities, Acta Arith. 111 (2004), 61-76.

[9] P. Fatou, Sur l'approximation des incommensurables et les series trigonometriques, C. R. Acad. Sci. (Paris) 139 (1904), 1019-1021.

[10] R. Goormaghtigh, L'Intermédiaire des Mathématiciens, 24 (1917), 88.

[11] I. Niven, H. S. Zuckerman and H. L. Montgomery, An Introduction to the Theory of Numbers, John Wiley \& Sons, Inc., New York, 1991.

[12] M. Le, On the Diophantine equation $\left(x^{3}-1\right) /(x-1)=\left(y^{n}-1\right) /(y-1)$, Trans. Amer. Math. Soc. 351 (1999), 1063-1074.

[13] M. Le, Exceptional solutions of the exponential Diophantine equation $\left(x^{3}-1\right) /(x-$ $1)=\left(y^{n}-1\right) /(y-1)$, J. Reine Angew. Math. 543 (2002), 187-192.

[14] M. Le, On Goormaghtigh's equation $\left(x^{3}-1\right) /(x-1)=\left(y^{n}-1\right) /(y-1)$, Acta Math. Sinica (Chin. Ser.) 45 (2002), 505-508.

[15] T. Nagell, The diophantine equation $x^{2}+7=2^{n}$, Ark. Mat. 4 (1961), 185-187.

[16] Yu. V. Nesterenko and T. N. Shorey On an equation of Goormaghtigh, Acta Arith. 83 (1998), 381-389.

[17] R. Ratat, L'Intermédiaire des Mathématiciens, 23 (1916), 150.

[18] A. Schinzel, On two theorems of Gelfond and some of their Applications, Acta Arith. 13 (1967/1968), 177-236.

[19] A. Schinzel, An improvement of Runge's theorem on diophantine equations, Comment. Pontificia Acad. Sci. 2 (1969), 1-9.

[20] T. N. Shorey, On the equation $a x^{m}-b y^{n}=k$, Nederl. Akad. Wetensch. Indag. Math. 48 (1986), 353-358.

[21] T. N. Shorey, Integers with identical digits, Acta Arith, 53 (1989), 187-205.

[22] C. L. Siegel, Approximation algebraischer Zahlen, Math. Z. 10 (1921), 173-213.

[23] C. L. Siegel, The integer solutions of the equation $y^{2}=a x^{n}+b x^{n-1}+\cdots+k$ (Under the pseudonym X), J. London Math. Soc. 1 (1926), 66-68.

[24] A. Thue, Über Annäherungswerte algebraischer Zahlen, J. Reine Angew. Math. 135 (1909) 284-305.

[25] R. T. Worley, Estimating $|\alpha-p / q|$, J. Austral. Math. Soc. Ser. A 31 (1981), 202-206.

[26] P. Z. Yuan, On the diophantine equation $\frac{x^{3}-1}{x-1}=\frac{y^{n}-1}{y-1}$, J. Number Theory 112 (2005), 20-25. 
Bo $\mathrm{He}$

Sichuan Provincial Key Laboratory of Computer Software

Sichuan Normal University

Chengdu, 610068

P. R. China

E-mail: hebo-one@hotmail.com, bhe@live.cn

Received: 6.9.2008.

Revised: 1.11.2008. 